\title{
Pharmacy Management of Vaccines
}

\author{
H. Eric Cannon, PharmD
}

\section{ABSTRACT}

BACKGROUND: Although standard vaccines have traditionally been granted full coverage in managed care, the recent introduction of several novel vaccine products has necessitated the revision of pharmacy management strategies throughout the nation.

OBJECTIVE: To review pharmacy management strategies for a number of emerging vaccines, with unique plan perspectives from SelectHealth, an Intermountain Healthcare company serving approximately 500,000 members in Utah.

SUMMARY: Because several recently introduced vaccines target previously unaddressed diseases and carry higher costs than traditional vaccines, several plans have adapted a novel approach to manage vaccine coverage on an individual product basis. At SelectHealth, recently introduced vaccines for rotavirus, respiratory syncytial virus (RSV), herpes zoster, and human papillomavirus (HPV) have required special attention in terms of pharmacy management. After carefully weighing acquisition and administration costs, anticipated uptake and use, direct and indirect health care costs averted, and quality of life issues, plan leadership decided to cover many of the new vaccines (i.e., rotavirus, RSV, and herpes zoster) under a nonstandard vaccination benefit. However, because substantial cost savings and high use of the quadrivalent HPV vaccine was anticipated within SelectHealth, the plan decided to fully cover the product.

CONCLUSION: Although they complicate traditional pharmacy management, novel vaccines provide clinical benefit that managed care organizations cannot ignore. One universal strategy will not suffice in managing all the different vaccines entering the market, and a tailored approach should be employed based on the individual characteristics and use of each product.

KEYWORDS: Vaccine, Pharmacy \& therapeutics; Herpes zoster, Rotavirus, RSV, HPV

J Manag Care Pharm. 2007;13(7)(suppl S-b):S7-S11

Copyright@ 2007, Academy of Managed Care Pharmacy. All rights reserved.

\section{Author}

H. ERIC CANNON, PharmD, is director of pharmacy and director of Health and Wellness, SelectHealth, Intermountain Healthcare, Salt Lake City, Utah.

AUTHOR CORRESPONDENCE: H. ERIC CANNON, PharmD, Director of Pharmacy and Director of Health and Wellness, SelectHealth/Intermountain Healthcare, 4646 W. Lake Park Blvd., Salt Lake City, UT 84130. Tel: (801) 442-5352; Fax: (801) 442-3006; E-mail: Eric.Cannon@selecthealth.org $\square$ verall, the U.S. Food and Drug Administration (FDA)-approved vaccines in use today are known to be safe and effective agents, and immunizations play a key role in the management of diseases with previously significant morbidity and mortality in the United States and worldwide. Still, more than 100,000 people (primarily adults) are affected by a vaccine-preventable disease per year in the United States alone. ${ }^{1}$ This apparent inconsistency indicates a need for improved vaccine management in the United States, and this need has only become more pressing in the advent of emerging vaccines for previously untargeted diseases and of combination products or vaccines employing novel delivery technologies. These factors have created an environment in which new vaccine management strategies are necessary to combat preventable disease.

As a framework for vaccine management in managed care organizations (MCOs), the National Committee for Quality Assurance's Health Plan Employer Data and Information Set (HEDIS) immunization measures provide an ideal starting point. Currently, 2 different measures exist: 1 for children and 1 for adolescents. ${ }^{2}$ For children, the measure accounts for the percentage of individuals who turned age 2 during the measurement year and who received the following vaccinations by their second birthday: 4 doses DTP (diphtheria-tetanus-pertussis), 3 doses OPV or IPV (polio), 1 dose MMR (measles-mumpsrubella), 3 doses HIB (Haemophilus influenzae type B), 3 doses hepatitis B, 1 dose VZV (varicella, or chicken pox), and 4 doses pneumococcal conjugate (meningitis and blood infections). For adolescents, the measure accounts for enrolled patients who received the following vaccinations by their 13th birthday: 2 doses MMR, 3 doses hepatitis B, and 1 dose VZV. ${ }^{2}$

Although improvement in immunization rates has been observed since the institution of the HEDIS measures in 1999, a quality gap still remains in the United States, with more than $20 \%$ of children and approximately $50 \%$ of adolescents missing 1 or more recommended immunizations. ${ }^{2}$ This lag in recommended immunization performance demonstrates a significant area for improvement in managed care. The emergence of new vaccines indicated for use in childhood or adolescence, such as those for rotavirus or human papillomavirus (HPV), only serves to complicate the issue further and necessitate additional vaccine management interventions by MCOs.

\section{New Vaccine Issues}

One MCO, SelectHealth, an Intermountain Healthcare company, has experienced changes in vaccine use and spending in the wake of a number of new vaccines being introduced to the market. At this commercial plan serving approximately 500,000 members in Utah, total vaccine spending averages $\$ 2.0$ to $\$ 2.4$ million per quarter, with an approximate $25 \%$ increase during 


\begin{tabular}{|c|c|c|}
\hline Input & Low & High \\
\hline Deaths & 20 & 70 \\
\hline Hospitalizations & 55,000 & 70,000 \\
\hline ED visits & 205,000 & 272,000 \\
\hline Office visits & 410,000 & 410,000 \\
\hline $\begin{array}{l}\text { Hospitalization } \\
\text { Length of stay }\end{array}$ & $\begin{array}{l}2.4 \text { days } \leq 1 \text { year } \\
2.0 \text { days }>1 \text { year }\end{array}$ & 3 days \\
\hline Work days missed & $\begin{array}{c}5 \text { days for } \\
\text { hospitalization/death } \\
2 \text { for others }\end{array}$ & $\begin{array}{c}5 \text { days for } \\
\text { hospitalization/death } \\
3 \mathrm{ED} ; 2 \text { for others }\end{array}$ \\
\hline
\end{tabular}

$C D C=$ Centers for Disease Control and Prevention; $E D=e m e r g e n c y$ department.

flu season. In terms of vaccination trends within the plan, use of Tdap (tetanus, diphtheria, acellular pertussis) has increased significantly, essentially replacing $\mathrm{Td}$ (tetanus, diphtheria) in light of the reemergence of pertussis (whooping cough) in the general population. In addition, anticipated adult vaccination recommendations for health care workers and those with young children are likely to further increase use of Tdap.

Another vaccine experiencing increased use at SelectHealth, despite issues surrounding limited supply, is ProQuad, the combination vaccine for MMR and varicella. Although the increased cost of this combination product would be expected to increase plan spending, the injection-reducing regimen of the product makes increased use of the MMR/varicella vaccine an essentially cost-neutral trend. Other recently introduced vaccines, such as those for HPV (Gardasil), rotavirus (RotaTeq), and herpes zoster (Zostavax), have experienced slower uptake than expected at SelectHealth. A discussion of the impact and management of emerging vaccines for these and other diseases in managed care follows, with direct experience from SelectHealth's plan perspective.

Rotavirus. Rotavirus-related gastroenteritis is associated with approximately $\$ 250$ million to $\$ 300$ million in direct costs and $\$ 700$ million to $\$ 900$ million in indirect costs in the United States. ${ }^{3}$ Estimates place the number of nationwide deaths associated with rotavirus-related gastroenteritis at approximately 20 and 70 on the low and high ends, respectively. In addition to causing an estimated maximum of 70,000 hospitalizations, 272,000 emergency department (ED) visits, and 410,000 office visits, the disease is associated with up to 5 missed work days per year per infected patient (Table 1).4,5

In the Rotavirus Efficacy $\&$ Safety Trial (REST), the rotavirus vaccine demonstrated efficacy in reducing health care use and work loss, compared with placebo. ${ }^{6}$ Most prominently, the vaccine offered a $95.8 \%$ rate reduction in the number of hospitalizations due to rotavirus-related gastroenteritis. ED visits, office visits, and work loss days also experienced rate reductions with the vaccine, compared with placebo (93.7\%, $86.0 \%$, and $86.6 \%$, respectively). ${ }^{6}$

With an eligible population of 12,300 at SelectHealth and a relatively high uptake (80\%) of the orally administered rotavirus vaccine, the plan would expect to spend approximately $\$ 2.6$ million per year on vaccination. Direct annual medical cost savings attributed to the vaccination of SelectHealth members against rotavirus are estimated to be about $\$ 90,000$ to $\$ 135,000$. Although minimal savings from vaccine administration would be realized, based on modeling, vaccine administration costs would mitigate societal costs by approximately $\$ 265$ per patient per year in terms of missed work days, inconvenience, and use of health care services.

As savings to the plan are projected to be minimal and the vaccine's benefit is likely to be largely societal, SelectHealth established a new benefit line termed a "nonstandard vaccination" to cover the rotavirus vaccine. With the application of regular medical coinsurance, the plan member is responsible for $20 \%$ of the cost of the vaccine; the rest is covered by SelectHealth. Rotavirus vaccine coverage policies are explained to patients during dialogue with their providers, at which time the providers can offer a recommendation on whether the patient should receive the vaccine. Weighing their portion of the cost of the vaccine against providers' recommendations allows the patients to make an educated decision on immunization against rotavirus. Also playing a part in SelectHealth's decision to cover the rotavirus vaccine was the fact that plan decision makers anticipated a recommendation from the Advisory Committee on Immunization Practices (ACIP) that all pediatric patients be immunized, although no such recommendation existed at the time coverage was instituted.

Respiratory syncytial virus (RSV). In healthy adolescents and adults, RSV usually causes severe colds with limited complications; however, in infants, infection with the virus can lead to serious respiratory complications. As the RSV vaccine, palivizumab (Synagis), has been on the market for several years, use within SelectHealth has been established as a seasonal phenomenon. Approximately $\$ 1$ million is spent per quarter during the season in which use of the vaccine is highest. The RSV season typically runs from November through April.

To effectively manage use of palivizumab within the plan, the pharmacy department at SelectHealth collaborated with plan neonatologists and pediatricians to establish a measure indicating the beginning of the RSV season. A decision was made to open the RSV season when there were 5 positive tests for the virus within the system during any given week (typically, sometime in November). Testing data are collected and maintained by Intermountain Laboratory Services at Primary Children's Hospital in Salt Lake. Infectious disease specialists at Primary Children's Hospital monitor collected data. 
Once the RSV season starts, SelectHealth notifies pediatricians that the RSV season has begun and that their authorizations for palivizumab are approved. Pediatricians can then begin vaccinating their patients. The RSV season is closed when there are fewer than 5 positive tests for the virus within the system during any given week. The goal of this protocol is to maintain use of the palivizumab as close to the 5 recommended doses as possible.

As with SelectHealth's policy with the rotavirus vaccine, the plan covers palivizumab under the nonstandard vaccination benefit, and patients are responsible for $20 \%$ of the cost of the vaccination. This policy was enacted because it was discovered that parents could take several steps to significantly reduce their children's exposure to RSV, and the burden of $20 \%$ of the vaccination cost encouraged them to take a more active role in preventing the disease.

HPV. HPV types 16 and 18 are implicated in 70\% of cervical cancer, of which more than 12,000 cases are diagnosed in the United States every year. The quadrivalent HPV vaccine was approved in June 2006 and targets these HPV types as well as types 6 and 11, which are implicated in 90\% of genital wart cases. The vaccine was evaluated in 4 randomized, placebocontrolled, double-blind clinical trials $(\mathrm{N}=15,719)$, with a median follow-up of 2 to 4 years, in concurrent administration with hepatitis $B$ vaccine $(n=1,871)$ and in combination with hormonal contraceptives ( $\mathrm{n}=13,293)$.

The quadrivalent HPV vaccine is indicated for immunization in females aged 9 to 26 years and is administered as a 3-dose injection series. ACIP recommends routine vaccination of patients aged 11 to 12 years (starting as early as 9 years, based on provider discretion), in sync with their current pre-middle school vaccination schedule, and "catch-up" injections are recommended for patients aged 13 to 26 years. Vaccination of male patients has not been studied and is not recommended by ACIP.

The cost of the quadrivalent HPV vaccine (average wholesale price [AWP] minus 10\%) is $\$ 140$ per dose, comprising $\$ 134$ in vaccine cost plus a $\$ 6$ administration fee. At SelectHealth, approximately 72,000 plan members are eligible for the vaccine, including 9,000 in the 11- to 12-year-old group and 63,000 in the 13- to 26-year-old "catch-up" group. The estimated uptake in vaccination in the group of 11- to 12-yearold plan members is initially slow, at $6 \%$ in the fall of 2006 , rising a steady state of $40 \%$ to $60 \%$ by 2009 . Breaking this down in terms of cost, SelectHealth projected a cost of $\$ 228,000$ for the vaccine in the fall of 2006, building to steady state at approximately $\$ 1.52$ to $\$ 2.27$ million in 2009 . In consideration of the initially larger "catch-up" population at SelectHealth, cost estimates for the plan were approximately $\$ 800,000$ in 2006, with 2007 and 2008 estimates being just more than $\$ 3$ million. Assuming that nearly all of the "catch-up" within the plan will take place within 2007 and 2008, the cost estimates within this

\begin{tabular}{l|c|c}
\hline \multicolumn{1}{c}{ TABLE 2 } & $\begin{array}{c}\text { Estimated Annual Savings Associated } \\
\text { With the Quadrivalent HPV Vaccine* }\end{array}$ \\
\hline Disease State & $\begin{array}{c}\text { Estimated No. } \\
\text { of Cases }\end{array}$ & $\begin{array}{c}\text { Estimated Total } \\
\text { Avoidance (\$) }\end{array}$ \\
\hline Cervical cancer & 15 & 478,548 \\
\hline CIN 2/3 & 447 & 1.10 million \\
\hline CIN 1 & 325 & 356,815 \\
\hline $\begin{array}{l}\text { Abnormal Pap test/ } \\
\text { no CIN }\end{array}$ & 2,973 & 711,175 \\
\hline \begin{tabular}{l} 
Genital warts \\
\hline Total
\end{tabular} & 297 & 135,918 \\
\hline * Possible annual vaccine cost savings: fewer treated for CIN 1/2/3, AIS, cancer \\
(assume FULL vaccination) \\
CIN=cervical intraepithelial neoplasia. \\
\hline
\end{tabular}

population will likely drop to zero by 2009 .

For the bottom-line cost of the quadrivalent HPV vaccine for SelectHealth, the estimates remain at approximately $\$ 1$ million for 2006, more than $\$ 4$ million for 2007 and 2008, and between $\$ 1.52$ and $\$ 2.27$ million once use hits steady state in 2009. In terms of total costs surrounding cervical cancer, it remains uncertain whether screening (i.e., pap tests) will continue in the long run at SelectHealth in light of the new vaccine. However, the continuation of screening is very likely, considering that the vaccine will not prevent all cases of cervical cancer. Still, the vaccine is estimated to save the plan approximately $\$ 2.7$ million per year at steady state, assuming full vaccination (Table 2 ).

When plan decision makers considered these substantial cost savings at steady state and the anticipated high use of the quadrivalent HPV vaccine in light of the demographics of SelectHealth's population, they decided to fully cover the vaccine for the remainder of 2006 and provide budgeting to allow for full coverage in the future. In other words, as opposed to the nonstandard vaccination benefit decision made with the rotavirus vaccine and palivizumab, SelectHealth plan leaders opted to make the quadrivalent HPV vaccine available to appropriate members at standard immunization status.

Although this decision was fairly straightforward at SelectHealth, considerations in the coverage of HPV immunizations in the future will likely be clouded by the emergence of new vaccines, the most prominent being the anticipated release of a bivalent HPV vaccine (Cervarix) within the next year. As new HPV vaccines become available, a new set of criteria will need to be evaluated to manage the products and provide the most clinically sound and cost-effective options to plan members. Among these criteria will be clinical considerations, such as the activity of new vaccines against different HPV types, the indications of the new vaccines, and any trial data available, with head-to-head data being the most preferable. 
TABLE 3 Efficacy of the Herpes Zoster Vaccine in the Shingles Prevention Study?

\begin{tabular}{|c|c|c|c|c|c|c|c|}
\hline Age (Years) & $\begin{array}{c}\text { No. of Zostavax } \\
\text { Subjects }\end{array}$ & No. of HZ Cases & $\begin{array}{c}\text { Incidence per } \\
\text { 1,000 Persons/ } \\
\text { Year }\end{array}$ & $\begin{array}{c}\text { No. of Placebo } \\
\text { Subjects }\end{array}$ & No. of HZ Cases & $\begin{array}{c}\text { Incidence per } \\
\text { 1,000 Persons/ } \\
\text { Year }\end{array}$ & $\begin{array}{c}\text { Efficacy (\%) } \\
(95 \% \text { CI) }\end{array}$ \\
\hline Overall & 19,254 & 315 & 5.4 & 19,247 & 642 & 11.1 & $\begin{array}{c}51 \\
(44-58) \\
\end{array}$ \\
\hline $60-69$ & 10,370 & 122 & 3.9 & 10,356 & 334 & 10.8 & $\begin{array}{c}64 \\
(56-71) \\
\end{array}$ \\
\hline $70-79$ & 7,621 & 156 & 6.7 & 7,559 & 261 & 11.4 & $\begin{array}{c}41 \\
(28-52) \\
\end{array}$ \\
\hline$>80$ & 1,263 & 37 & 9.9 & 1,332 & 47 & 12.1 & $\begin{array}{c}18 \\
(29-48)\end{array}$ \\
\hline
\end{tabular}

Notes: Mean duration of follow-up: 3.1 years.

Primary efficacy analysis: 30 days postvaccination $\mathrm{HZ}$ development.

Highest efficacy in 60-to-69-year olds, lesser efficacy in older patients.

$\mathrm{CI}=$ confidence interval; $\mathrm{HZ}=$ herpes zoster.

Other plan-specific considerations at SelectHealth in light of new HPV vaccines becoming available will include the current use of the quadrivalent HPV vaccine, the plan's ability to contract for the different products, and the difficulty surrounding integration of new products. These factors become increasingly important because SelectHealth/Intermountain Healthcare is an integrated system.

Herpes zoster. The single-dose herpes zoster vaccine (Zostavax) was approved in May 2006 for the prevention of postherpetic neuralgia (PHN, i.e., shingles) in patients aged 60 years or older. One unique issue in the recent consideration of the herpes zoster vaccine in Intermountain's hospital pharmacy and therapeutics committees is that the product is a live-virus vaccine. Hospitalists raised concerns about using a potentially infectious agent around immunocompromised patients. This issue may hold specific implications for integrated systems. Still, PHN was one area in which plan leaders felt there was a significant opportunity for cost savings and overall benefit to the plan population.

In the Shingles Prevention Study, the herpes zoster vaccine demonstrated the greatest clinical benefit in patients aged 60 to 69 years, with $64 \%$ efficacy (Table 3). ${ }^{8}$ In patients developing PHN, the mean duration of clinically significant pain (greater than 3 on a 10-point scale) was 20 days in the vaccine group versus 22 days in the placebo group. Overall, the vaccine was $39 \%$ efficacious against PHN in patients who developed herpes zoster after vaccination. In addition, zoster-related complications were similar in the vaccinated and placebo groups. ${ }^{8}$

The cost of the herpes zoster vaccine (AWP minus 10\%) is approximately $\$ 178$, including the administration fee. At SelectHealth, which has a generally younger population, approximately 21,000 patients are eligible for the vaccine, but the overall uptake is expected to be low, with higher uptake during flu vaccination season. Total costs to the plan are expected to be $\$ 112,000$ initially with low uptake (3\%), ramping up to $\$ 299,000$ at steady state uptake (8\%) in 2009.

For the financial implications of the vaccine, there was $\$ 388,000$ in herpes zoster-related costs at SelectHealth in 2005 . With an assumption of $50 \%$ immunization with the herpes zoster vaccine and 64\% efficacy, the potential savings are as much as $\$ 124,000$. The bottom-line cost of the herpes zoster vaccine to SelectHealth is estimated to be $\$ 240,000$ to $\$ 300,000$ annually, but this cost could be reduced if the coverage required member coinsurance and/or a deductible. For example, 20\% coinsurance would reduce plan costs by $\$ 60,000$ annually. Taking this into consideration, along with a relatively low expected uptake of the vaccine, decision makers at SelectHealth opted to make the herpes zoster vaccine available to plan members under a nonstandard immunization benefit, applying a $20 \%$ coinsurance requirement.

\section{Conclusion}

Although they complicate traditional pharmacy management, novel vaccines provide clinical benefit that MCOs cannot ignore. Furthermore, with several new vaccines on the market and even more on the horizon, the management of vaccines within managed care is of increasing importance. Although vaccine coverage was relatively unmanaged in the past, tremendous opportunities exist now in the immunization arena for MCOs to provide cost-effective, clinical benefits to their plan members.

Clearly, in the case of SelectHealth, benefit design has been critical to the management of vaccines within the plan population. Plan decision makers have taken many components into consideration, including clinical, economic, and governmental factors, and changes have been made accordingly to Select Health's existing benefit design. 
Obviously, a single strategy will not suffice in managing all the different vaccines entering the market, and a tailored approach should be employed based on the individual characteristics and use of each product. Each vaccine management strategy available to managed care stakeholders has inherent strengths and weaknesses. The nonstandard vaccination strategy employed at SelectHealth, in particular, relies heavily on plan physicians to discuss with their patients the benefits and disadvantages of a specific vaccine. Decision makers at SelectHealth acknowledge that, ultimately, other vaccination strategies will need to be employed. Emerging vaccines will require plan leaders to continually revisit SelectHealth's benefit design to provide clinically and economically sound immunization options to its members.

\section{DISCLOSURES}

This article is based on a presentation given by the author at a symposium, "The Value of Preventive Medicine: A Look at Vaccine Management," held April 11, 2007, at the Academy of Managed Care Pharmacy's 19th Annual Meeting and Showcase in San Diego, California. The symposium was supported by an educational grant from GlaxoSmithKline. The author discloses that he has received an honorarium for participation in the presentation and in this supplement. He discloses no potential bias or conflict of interest relating to this article.

\section{REFERENCES}

1. Poland GA. Standards for adult immunization practices. Am J Prev Med. 2003;25(2):144-50

2. National Committee on Quality Assurance. State of health care quality. 2006 Available at: http://www.ncqa.org/Communications/SOHC2006/SOHC_2006. pdf. Accessed March 20, 2007.

3. Centers for Disease Control and Prevention. CDC's advisory committee recommends new vaccine to prevent rotavirus. Press release. February 21, 2006. Available at: http://www.cdc.gov/od/oc/media/pressrel/r060221.htm. Accessed July 2, 2007.

4. Velazquez FR, Matson DO, Calva JJ. Rotavirus infection in infants as protection against subsequent infections. N Engl J Med. 1996;335:1022-28.

5. Parashar U, Bresee JS, Gentsch JR, Glass RI. Rotavirus. Emerg Infect Dis. 1998;4(4). Available at: http://www.cdc.gov/ncidod/EID/vol4no4/parashar.htm. Accessed July 2, 2007

6. Lee BP, Staat M, Louie L. Nonmedical costs associated with rotavirus disease. Pediatr Infect Dis J. 2005;24:984-88.

7. Vesikari T, Matson DO, Dennehy P. Safety and efficacy of a pentavalent human bovine (WC3) reassortment rotavirus vaccine. N Engl J Med. 2006;354(1):23-33.

8. Oxman MN, Levin MJ, Johnson GR. A vaccine to prevent herpes zoster and postherpetic neuralgia in older adults. N Engl J Med. 2005;352:2271-84. 